\title{
Cortical Auditory Disorder Caused by Bilateral Temporal Infarctions
}

\author{
Kazuhiro IshII, Yuji UedA, Norio OHKoshi, Hidehiro Mizusawa and Shin'ichi ShoJI
}

\begin{abstract}
We present a 55-year-old man who suddenly became afflicted with a bilateral auditory disturbance. Auditory acuity was preserved relatively well. Brainstem auditory evoked potentials (BAEPs) were normal. Middle latency auditory evoked potentials (MLAEPs) revealed the total absence of all waves. Brain computed tomography (CT) showed infarctions of the temporal lobes bilaterally, involving the superior temporal and transverse temporal gyri. The recognition of verbal and nonverbal sounds was also impaired. Brain CT, MLAEPs and auditory recognition tests were useful in diagnosing the cortical auditory disorder.
\end{abstract}

(Internal Medicine 34: 801-805, 1995)

Key words: verbal sound, nonverbal sound, middle latency auditory evoked potentials

\section{Introduction}

Cortical auditory disorder is a rare condition defined by an impaired capacity to recognize both verbal and nonverbal sounds despite adequate hearing ability (1-4). This disorder is thought to be distinct from auditory agnosia in a restricted sense, in which nonverbal or environmental sounds are selectively impaired, and pure word deafness, in which verbal sounds are selectively impaired (4). Some reports have proposed methods to discriminate between these disorders using middle latency auditory evoked potentials (MLAEPs) $(5,6)$ and imaging studies $(3,7-9)$.

We report a patient with a cortical auditory disorder caused by bilateral temporal lesions, and examine the results of verbal and nonverbal sound recognition tests in detail.

\section{Case Report}

This 55-year-old right-handed man suddenly suffered a loss of consciousness and a mild left hemiparesis on January 4, 1994, for which he was admitted to a nearby hospital. A few days later, he became alert and recovered from his left hemiparesis. However, he did not appropriately respond to verbal questions and environmental sounds. He could correctly understand writing commands. Computed tomography (CT) of the brain revealed a new embolic lesion in the right temporoparietal area and a left temporal infarct that he had suffered years previously. He complained of a bilateral auditory distur- bance and was transferred to our hospital for further evaluation.

His past medical history was significant for a cerebral embolism of his left temporal lobe in 1979 and a complete atrioventricular block that had been treated with an implantable pacemaker. He completely recovered from his right hemiparesis and dysarthria within a few months. Subsequently, he continued working as a truck driver.

On admission on February 3, 1994, physical examination revealed an alert man with a normal blood pressure (116/70 $\mathrm{mmHg}$ ), and a regular pacing rhythm $(72 / \mathrm{min})$. He demonstrated a cooperative attitude on examination, replying with a loud voice and did not say more than necessary. Neurological examination revealed bilateral auditory impairment with no deviation on the Weber test. He also manifested a left central hemifacial palsy. Deep tendon reflexes were slightly active bilaterally with negative pathological reflexes. He had normal visual fields with sufficient visual acuity and normal muscle tonus and power. There was no unilateral spatial neglect, visual agnosia, apraxia, sensory disturbance, ataxia, or other neurological abnormality.

Laboratory findings, including a peripheral blood cell count, serum biochemical analysis and urinalysis all were normal. A chest radiograph revealed mild cardiomegaly. Electrocardiography revealed a normal regular pacing rhythm. Neither an atrial thrombus nor a valvular abnormality was observed on an echocardiogram. Cerebral computed tomography (CT) demonstrated cortical and subcortical infarctions in the temporal lobes bilaterally. These infarctions involved the posterior part of the

From the Department of Neurology, Institute of Clinical Medicine, University of Tsukuba, Tsukuba

Received for publication November 8, 1994; Accepted for publication May 5, 1995

Reprint requests should be addressed to Dr. Kazuhiro Ishii, the Department of Neurology, Institute of Clinical Medicine, University of Tsukuba, Tenoudai Tsukuba, Ibaraki 305 
transverse temporal (Heschl's) gyri and the superior temporal gyrus. The brainstem and medial geniculate bodies were intact (Fig. 1).

\section{Higher function tests}

On the Wechsler adult intelligence scale-revised (WAIS-R), his verbal, performance, and total intelligence quotients (IQs) were 83,85 , and 84 , respectively. Short- and long-term memory, examined using the Wechsler memory scale-revised, were completely normal. On the Token test presented as written commands, 96\% (159/165) of the subject's responses were correct. The results on the Western aphasia battery (WAB) were: spontaneous speech, $8 / 10$; auditory verbal comprehension, $0.5 / 10$; repetition, $0 / 10$; naming, $7 / 10$; reading, $6 / 10$; and writing, 1/10. A mild sensory aphasia was detected using this battery. The WAB also disclosed that the examination items presented as auditory verbal commands elicited extremely poor results and very slight paralexia and paragraphia were revealed in the items of reading and writing, respectively. However, it was not difficult for him to understand any of the examination items presented as written commands.

\section{Acoustic tests and evoked responses}

Otological examination revealed an old left middle otitis from before. Acoustic reflexes were elicited at normal thresholds. Ipsilateral and contralateral stapedius reflexes were present. Subsequently, standard pure-tone threshold audiometry demonstrated a sensorineural hearing loss pattern on the 50th disease day. This hearing loss had recovered partially by the 85 th disease day (Fig. 2). Conventional speech audiometry tests were performed on the 50th and the 85th disease days. However, he could not discriminate any of the stimuli bilaterally at $80 \mathrm{~dB}$ on either day. All brainstem auditory evoked potential (BAEP) components from I to VII were recorded bilaterally with normal latency. MLAEPs were totally absent for all components, including $\mathrm{Po}$, No and $\mathrm{Na}$, and the late components, $\mathrm{Pa}$ and $\mathrm{Nb}$ (Fig. 3) (10).

\section{Auditory recognition tests}

Matching verbal-verbal sound tasks (auditory discrimina-
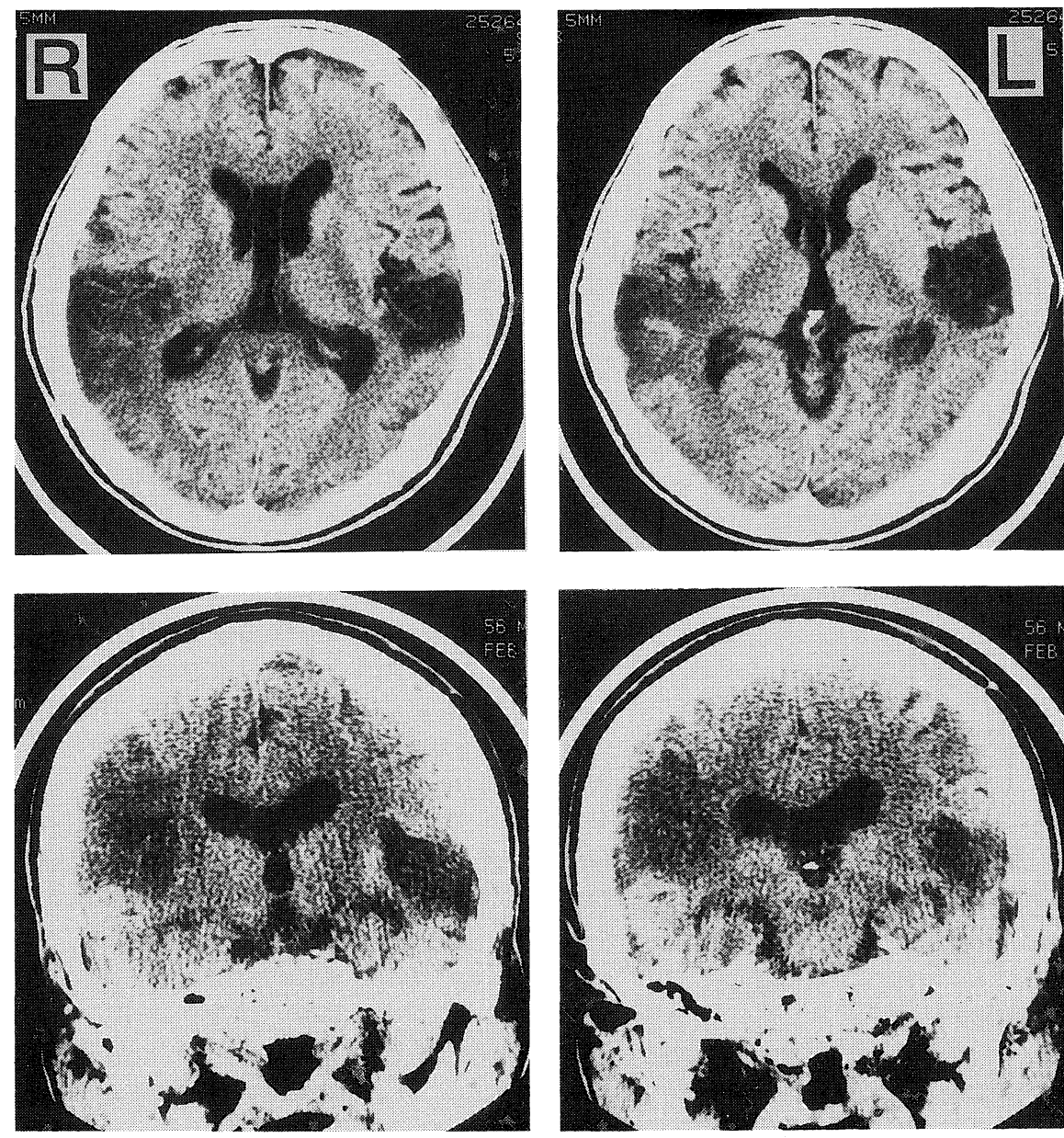

Figure 1. Brain computed tomography (CT) images. Axial (upper) and coronal (lower) views demonstrating a large right temporo-parietal infarct and an old left temporal lobe infarction, including the transverse temporal (Heschl's) and superior temporal gyri. 
$(\mathrm{dB})$

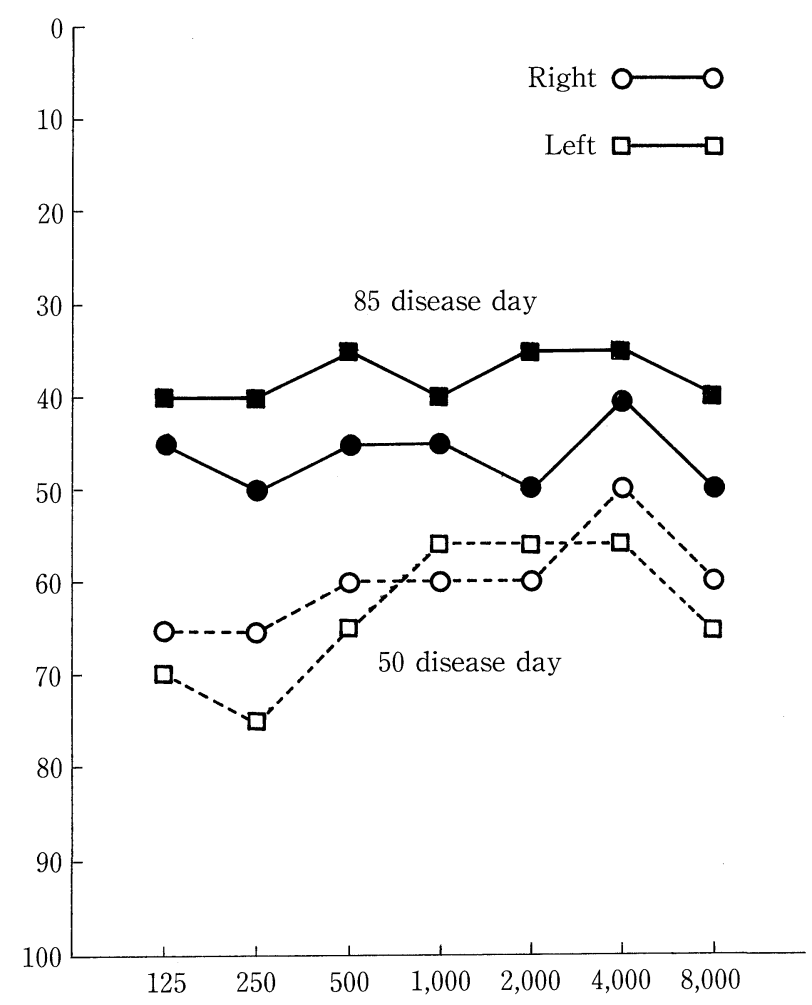

Figure 2. Audiogram performed on the 50th (broken line) and the 85th (solid line) disease days. The square and circle represent records for the right ear and left ear, respectively.

tion between two words) were impaired (correct answers 13/ 32). Matching nonverbal-nonverbal sound tasks (discrimination of whether two environmental sounds are the same or different) also were impaired (correct answers 12/32). Pointing word tasks involving nonverbal sounds were impaired (correct answers 14/32). Pointing word tasks involving nonverbal sounds were as follows: 1 ) Sets of four cards were shown to the subject, each with a word describing an environmental sound or an unrelated word. 2) Specifically, the words on each set of four cards included the correct sound, an acoustically related sound, a semantically related sound and an unrelated word. 3) The subject listened to prerecorded environmental sounds and pointed to the card corresponding to the sound. He could not identify nonverbal sounds. For example, he answered that the sound of a dog crying sounded like a man speaking, and that the sound of rainfall sounded like a flowing stream. The subject's ability to imitate environmental sounds (being shown a card with a word describing an environmental sound, and then imitating the sound) was well-preserved (correct answers 23/24). Matching of music (discrimination between two sorts of music) was impaired (correct answers 5/12). He could not recognize familiar music by listening, nor sing songs at a normal tempo, melody, and rhythm as he had been able to before.

\section{Clinical course}

This patient experienced a cerebral reembolism during antiplatelet therapy, but without pacing failure. Warfarin administration ( $2 \mathrm{mg} /$ day) was started to prevent a third embolism. His motor function was well-preserved for daily life activities. Although verbal communication was possible by means of writing, his auditory disability in discriminating verbal and nonverbal sounds continued for half a year after the onset of symptoms.

\section{Discussion}

The auditory impairment in the present patient was attributed to "cerebral" lesions. The sudden onset of symptoms and neurologic deficits, the relatively good preservation of bilateral auditory acuity, the presence of bilateral stapedius reflexes and normal BAEPs, and the bilateral temporal infarcts on CT all suggested "cerebral" lesions. It was quite impossible to attribute this bilateral auditory disturbance to only the old, left otitis media. The auditory recognition tests revealed impaired recognition of both verbal and nonverbal sounds. Subsequently, we diagnosed this patient with a cortical auditory disorder (1, 4). The previous infarct in the left temporal lobe, and the subsequent infarct in the right temporo-parietal lobe, might have caused this cortical auditory disorder.

There is confusion in the terminology used to describe auditory disturbances of cerebral origin. Similar features of impaired sound recognition for verbal and nonverbal sounds have been reported using different terms, i.e. cortical deafness (5), cortical auditory disorder (1-3), generalized auditory agnosia $(7)$, auditory agnosia $(9,11)$ or auditory agnosia and pure word deafness (12). In fact, cortical auditory disorders and other disorders frequently evolve from a state of cortical deafness in which all types of auditory acuity are extremely impaired. Most cases with impairment of nonverbal sound recognition are accompanied by some degree of impairment in verbal sound recognition. For these reasons, it is often difficult to clearly discriminate among these disorders.

The failure in the matching and discriminating sound tests in regard to both nonverbal and verbal cues suggested the possibility of a perceptive impairment. We thought that this disorder was more consistent with a lower level of impairment than a cognitive impairment. The term "auditory agnosia" is inadequate to describe the present case with damage at the perceptive level. Thus, we labelled the present case, which was similar to the cases of Kanshepolsky et al (1) and others (2, 3), as "cortical auditory disorder".

Several studies have suggested that middle latency auditory evoked potentials (MLAEPs) are useful to differentiate between cortical auditory disorder and other disorders (5). MLAEPs of cortical auditory disorders are characterized by the total absence or absence of late components $(5,6)$, while patients with pure word deafness (13) and auditory agnosia (14) have been found to have almost normal components on MLAEPs. As the present case demonstrated no peaks on MLAEPs, the findings were compatible with a diagnosis of cortical auditory 

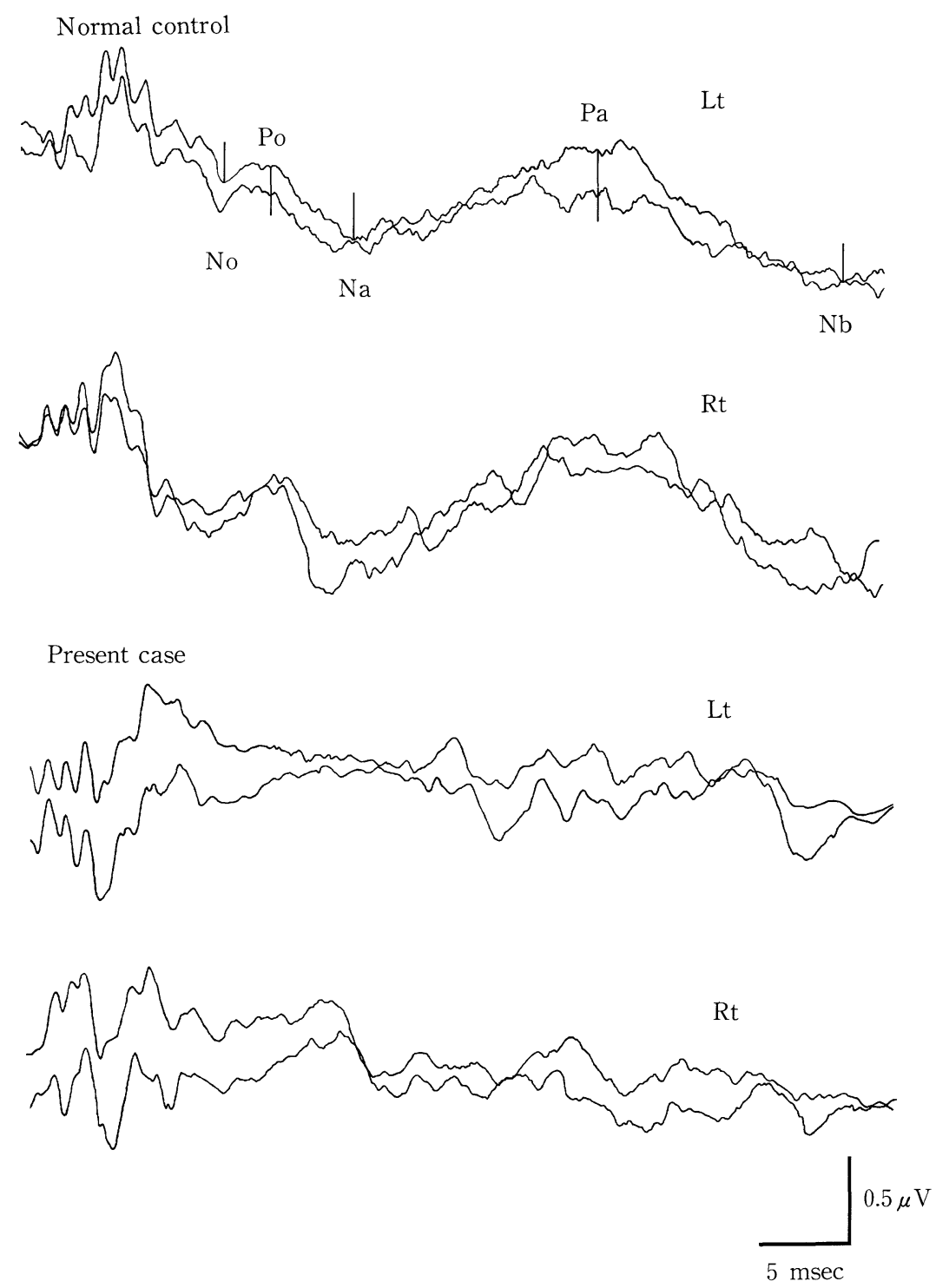

Figure 3. Middle latency auditory evoked potentials (MLAEPs) of a normal control (upper) and the present patient (lower). The MLAEPs of the present case reveal absence of all waves bilaterally.

disorder.

The causal lesions of cortical auditory disorder are variable $(1-3,9,11)$. Most of the cases investigated have been associated with lesions in the temporal lobes bilaterally, particularly in both posterior segments of the superior temporal and transverse temporal (Heschl's) gyri $(1,11)$. In a few cases, magnetic resonance imaging has localized the lesions to subcortical areas $(7,8)$. The lesions in the present case were in the posterior halves of both the superior temporal and transverse gyri, and in the right inferior parietal lobe and its subcortical portions. These lesions were similar to those described in Miceli's report (2).

The mechanism of this disorder and issues regarding termi- nology remain to be elucidated. The criteria for cortical auditory disorder should be defined in detail and a uniform battery of auditory recognition tests should be established in the future.

\section{References}

1) Kanshepolsky J, Kelley J, Waggener J. A cortical auditory disorder. Neurology 23: 699, 1973.

2) Miceli G. The processing of speech sounds in a patient with cortical auditory disorder. Neuropsychologia 20: 5, 1982.

3) Mendez MF, Geehan GR. Cortical auditory disorders: Clinical and psychoacoustic features. J Neurol Neurosurg Psychiatry 51: 1, 1988.

4) Heilman KM, Valenstein E. Clinical Neuropsychology. 3rd edition. Oxford University Press, New York, Oxford, 1993, p.241.

5) Michel J, Peronnet F, Schott B. A case of cortical deafness: Clinical and 


\section{A Case of Cortical Auditory Disorder}

electrophysiological data. Brain Lang 10: 367, 1980.

6) Jerger J, Weikers N, Sharbrough F, Jerger S. Bilateral lesions of the temporal lobe. A case study. Acta Otolaryngol (Stockh.) [suppl] 258: 1, 1969.

7) Kazui S, Naritomi H, Sawada T, Inoue N. Subcortical auditory agnosia. Brain Lang 38: 476, 1990.

8) Motomura N, Yamadori A, Mori E, Tamura F. Auditory agnosia: Analysis of a case with bilateral subcortical lesions. Brain 109: 379, 1986.

9) Rosati G, DeBastiani P, Paolino E, Prosser S, Arslan E, Artioli M. Clinical and audiological findings in a case of auditory agnosia. J Neurol 227: 21, 1982.

10) Özdamar Ö, Kraus N, Curry F. Auditory brain stem and middle latency responses in a patient with cortical deafness. Electroencephalogr Clin Neurophysiol 53: 224, 1982.

11) Oppenheimer DR, Newcombe F. Clinical and anatomic findings in a case of auditory agnosia. Arch Neurol 35: 712, 1978.

12) Goldstein MN, Brown M, Holander J. Auditory agnosia and word deafness: Analysis of a case with three-year follow up. Brain Lang 2: 324, 1975.

13) Albert ML, Sparks R, von Stockert T, Sax D. A case study of auditory agnosia: Linguistic and nonlinguistic processing. Cortex 8: 427, 1972.

14) AssalG, Despland PA. Presentation d'un cas d'agnosie auditive. Otoneuroophtalmologie 45: 353, 1973 (in French). 